Article

\title{
Speeding Up the Identification of Cystic Fibrosis Transmembrane Conductance Regulator-Targeted Drugs: An Approach Based on Bioinformatics Strategies and Surface Plasmon Resonance
}

\author{
Marco Rusnati ${ }^{1,+}$ (D), Davide Sala ${ }^{2,3,+}$, Alessandro Orro ${ }^{2,+}$, Antonella Bugatti ${ }^{\text {, }}$ \\ Gabriele Trombetti ${ }^{2}$, Elena Cichero ${ }^{3}$, Chiara Urbinati ${ }^{1}$, Margherita Di Somma ${ }^{1}$ (D), \\ Enrico Millo ${ }^{4,5}$, Luis J. V. Galietta ${ }^{6}$, Luciano Milanesi ${ }^{2}$, Paola Fossa ${ }^{3, *}$ and Pasqualina D'Ursi $^{2, *}$ \\ 1 Department of Molecular and Translational Medicine, University of Brescia, 25123 Brescia, Italy; \\ marco.rusnati@unibs.it (M.R.); antonella.bugatti@unibs.it (A.B.); chiara.urbinati@unibs.it (C.U.); \\ m.disomma@unibs.it (M.D.S.) \\ 2 Institute for Biomedical Technologies, National Research Council (ITB-CNR), 20090 Segrate, Italy; \\ d.sala1388@gmail.com (D.S.); alessandro.orro@itb.cnr.it (A.O.); gabriele.trombetti@gmail.com (G.T.); \\ luciano.milanesi@itb.cnr.it (L.M.) \\ 3 Department of Pharmacy, Section of Medicinal Chemistry, School of Medical and Pharmaceutical Sciences, \\ University of Genoa, 16132 Genoa, Italy; cichero@difar.unige.it \\ 4 Department of Experimental Medicine, Section of Biochemistry, University of Genoa, 16132 Genoa, Italy; \\ enrico.millo@unige.it \\ 5 Center of Excellence for Biomedical Research (CEBR), University of Genoa, 16132 Genoa, Italy \\ 6 Istituto Giannina Gaslini, 16147 Genoa, Italy; l.galietta@tigem.it \\ * Correspondence: fossa@difar.unige.it (P.F.); pasqualina.dursi@itb.cnr.it (P.D.); \\ Tel.: +39-010-3538238 (P.F.); +39-02-26422600 (P.D.) \\ + These authors contributed equally to this work.
}

Received: 24 October 2017; Accepted: 4 January 2018; Published: 8 January 2018

\begin{abstract}
Cystic fibrosis (CF) is mainly caused by the deletion of Phe $508(\Delta \mathrm{F} 508)$ in the cystic fibrosis transmembrane conductance regulator (CFTR) protein that is thus withheld in the endoplasmic reticulum and rapidly degraded by the ubiquitin/proteasome system. New drugs able to rescue $\triangle$ F508-CFTR trafficking are eagerly awaited. An integrated bioinformatics and surface plasmon resonance (SPR) approach was here applied to investigate the rescue mechanism(s) of a series of CFTR-ligands including VX809, VX770 and some aminoarylthiazole derivatives (AAT). Computational studies tentatively identified a large binding pocket in the $\triangle$ F508-CFTR nucleotide binding domain-1 (NBD1) and predicted all the tested compounds to bind to three sub-regions of this main pocket. Noticeably, the known CFTR chaperone keratin-8 (K8) seems to interact with some residues located in one of these sub-pockets, potentially interfering with the binding of some ligands. SPR results corroborated all these computational findings. Moreover, for all the considered ligands, a statistically significant correlation was determined between their binding capability to $\triangle$ F508-NBD1 measured by SPR and the pockets availability measured by computational studies. Taken together, these results demonstrate a strong agreement between the in silico prediction and the SPR-generated binding data, suggesting a path to speed up the identification of new drugs for the treatment of cystic fibrosis.
\end{abstract}

Keywords: cystic fibrosis; computational chemistry; molecular dynamics; molecular modeling; surface plasmon resonance 


\section{Introduction}

Cystic Fibrosis (CF) is the most common lethal monogenic disorder in Caucasians. It is due to different mutations in the cystic fibrosis transmembrane conductance regulator (CFTR), a protein composed of five domains: two nucleotide binding domains (NBD1 and 2), two transmembrane domains (MSD1 and 2) and one regulatory domain (R) [1]. The mutations causing CF are classified in five groups, according to the mechanism of CFTR loss-of-function [2]. The most common one, occurring in $70-90 \%$ of CF patients, is the deletion of phenylalanine $508(\Delta \mathrm{F} 508)$ in NBD1 that causes inappropriate folding and structural instability of $\triangle$ F508-CFTR that, for this reasons, remains trapped in the endoplasmic reticulum and is rapidly degraded by the ubiquitin/proteasome system.

Several molecular chaperones and co-chaperones, among which Hsp70/Hsc70, Hsp90, CHIP and BAG-2 [3] are involved in CFTR intracellular processing. In particular, keratin 8 (K8) plays an important role in $\triangle F 508-C T F R$ retention and degradation, through a preferential binding to $\triangle$ F508-CTFR NBD1 with respect to its wild type (WT) counterpart. Accordingly, the inhibition of $\Delta$ F508-CFTR/K8 interaction leads to the rescue of the defective $\triangle$ F508-CFTR processing [4,5]. However, also if the mutant protein is targeted to the plasma membrane through rescue maneuvers, the probability that its channel is open is reduced. The deficit of CFTR at the plasma membrane has a major impact on the respiratory system, since CF patients produce a thick mucus that cannot be cleared, resulting in an impairment of innate defense against bacteria [6]. The resulting pulmonary infections cause a progressive loss of respiratory function that, in the last stages of the disease, may require lung transplantation as a life-saving intervention $[7,8]$.

Current therapies are mostly aimed at treating CF symptomatically, e.g., by aggressive antibiotic strategies for the clinical management of bacterial lung infections. Although these therapies has significantly pushed forward the mean survival age of patients from early childhood in the 1950s to the late 30s at present [9], the burden of CF care remains very high and life quality and expectancy for most CF patients are still limited.

An ambitious therapeutic alternative is to address CF systemically, by means of small molecules that restore the trafficking (correctors) and gating (potentiators) capacity of mutated CFTR. At this regard, a big effort has been made in the last ten years to determine the complete human CFTR 3D structure by means of homology modelling, NMR and X-rays studies. In addition, computational studies and cell- or tissue-based assays have been used to elucidate the mechanisms of action of CFTR drugs, in particular VX809 [10-15].

In this context, the mutated CFTR protein and its NBD1 domain represent ideal targets for small molecules with rescuing activity on $\triangle$ F508-CFTR $[10,16]$. Leveraging high-throughput screening by companies and academic labs, this approach has already lead to some interesting compounds that however remain in need of better elucidation of their molecular mechanism of action. To this lack of knowledge surely contributes the fact that up to now, the available experimental structures of human WT or mutated NBD1 and complete CFTR are largely incomplete: as concerns NBD1, the human protein lacks some regions and/or contains solubilizing mutations (reviewed by Lewis and co-workers [17]). As for the recently released human CFTR model, its experimental structure presents unsolved portions [14], so that a detailed picture of the conformational changes which result in channel opening/closing remain to be fully resolved [18].

To clarify these points, possibly allowing a rational design and full characterization of potential CF drugs, here we exploited a synergistic approach including high performance computational studies of the murine NBD1 protein (available as complete WT and $\triangle 508 \mathrm{~F}$ mutated structures) and biomolecular interaction analysis by surface plasmon resonance (SPR) for an accurate validation of the in silico predictions. The former allows the full evaluation of the dynamic behavior(s) of proteins, simulating real biological events such as the molecular interactions between a ligand and its target and the conformational changes of CFTR variants in respect to the WT protein. The latter represents the golden standard label-free technology for real-time analysis of molecular interactions also in the field of $\mathrm{CF}$ 
drug discovery [8,19], being able to generate high-quality data on affinity, kinetic and mechanistic aspects of interactions occurring between target proteins and drug candidates [20,21].

To solidly validate the complementary approach made up by computational and SPR studies, here we have investigated the interaction of three well characterized and promising CF correctors and four new potentially interesting compounds (Figure 1) [22-26] and characterized their ability to bind to $\triangle$ F508-NBD1.<smiles>Cc1ccc(NC(=O)C2(c3ccc4c(c3)OC(F)(F)O4)CC2)nc1-c1cccc(C(=O)O)c1</smiles>

$1, \mathrm{VX809}$

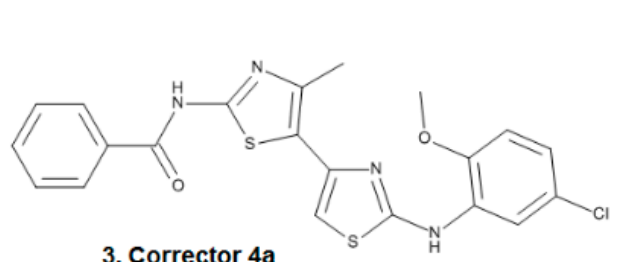

3, Corrector $4 a$

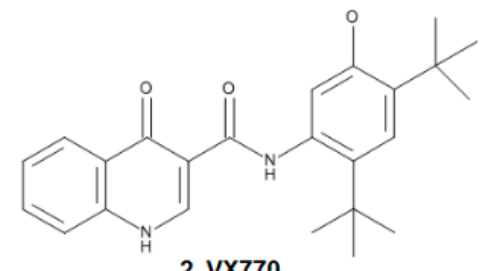

$2, \operatorname{vx770}$

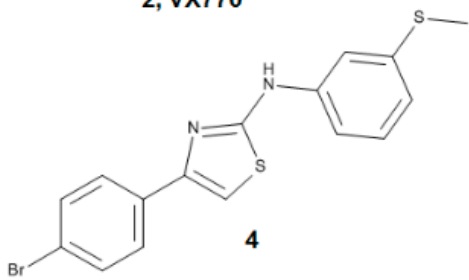

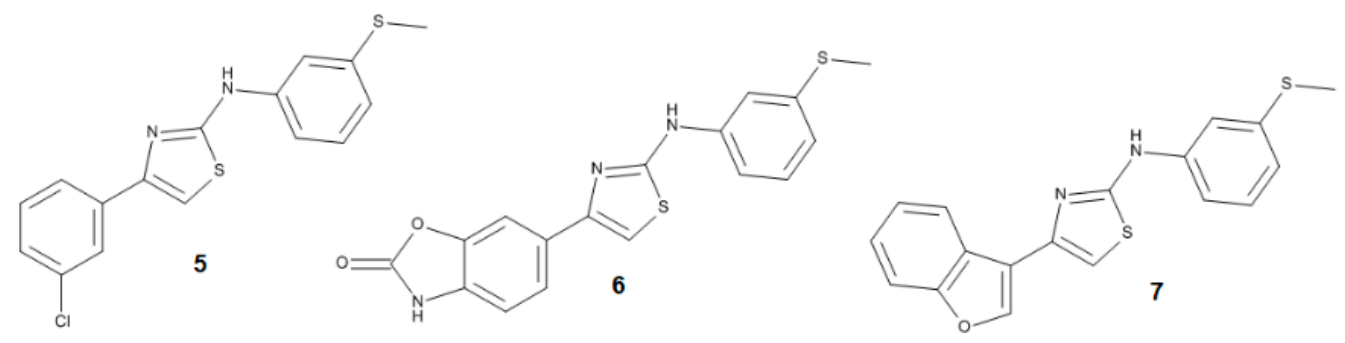

Figure 1. Structures of ligand dataset.

The results obtained by the computational approach resulted in agreement with SPR findings, providing a detailed characterization of the ligand-protein interaction useful for an efficient screening of large compound libraries, a better rational design and a tailored synthesis of new lead compounds for the rescue of mutated CFTR.

\section{Results}

\subsection{Characterization of the $\triangle F 508-N B D 1$ Flexibility by Molecular Dynamics Simulations (MDS)}

Several studies demonstrated that the $\triangle$ F508-NBD1 apo is thermodynamically and kinetically destabilized at physiological temperature in respect to the WT NBD1 apo [27-29]. In order to highlight which differences in structure and backbone flexibility were induced in the protein by the mutation, we firstly focused our attention on exhaustive MDS studies of $200 \mathrm{~ns}$ on both WT NBD1 and $\triangle$ F508-NBD1 forms. In agreement with literature data [30,31] a very high flexibility was observed for $\triangle$ F508-NBD1 in comparison to the WT NBD1, that was not limited to the close environment of the $\Delta$ F508 residue (Figure 2A).

To compare the flexibility of $\triangle \mathrm{F} 508$ and WT NBD1, we computed the root mean square fluctuation (RMSF), an average measure of atomic mobility of backbone atoms along the MDS. Results show very high flexibility (Figure 2A) in five loops contained in a large region that forms the binding pocket (predicted by CASTp program) and that includes residues from a highly conserved regions in all NDB proteins, such as the Walker B motif, the signature sequence LSGGQ, the Q- and the H-loops (Figure 2B) [32]. 

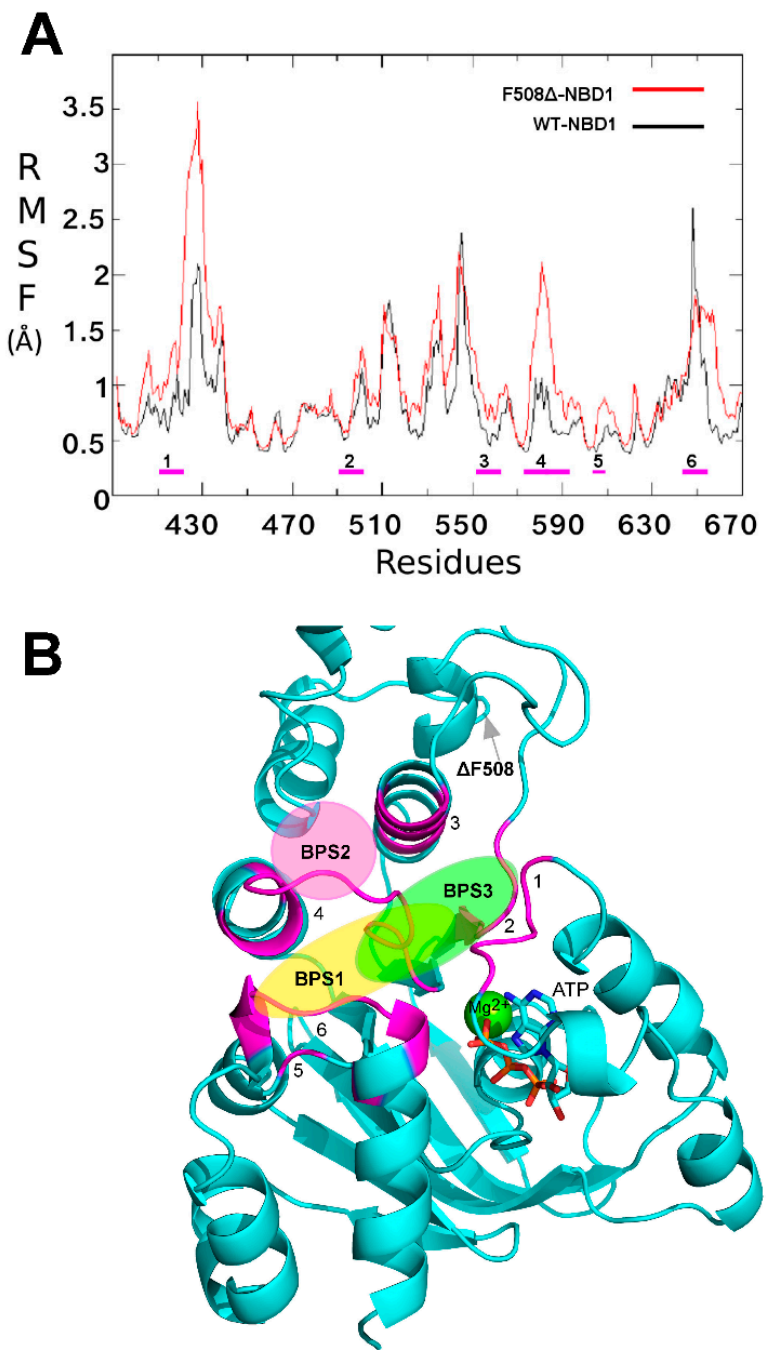

Figure 2. (A) Root mean square fluctuation (RMSF) of $\triangle$ F508-NBD1 protein. RMSF values for $\triangle$ F508 and WT NBD1 are reported in red and black respectively. Magenta bold dashes indicate the regions composing the main binding pocket, numbered from one to six; (B) Ribbon representation of the $\triangle$ F508-NBD1 protein. The regions forming the main binding pocket predicted by CASTp are highlighted in magenta and numbered from one to six. The three binding sub-pockets predicted for the data-set ligands are also indicated as colored circles: yellow, binding sub-pocket 1 (BSP1), the putative binding site for VX809; magenta, BSP2, the putative binding site for compound 5; light green, BSP3, the putative binding site for compounds $\mathbf{4}$ and $\mathbf{6}$. The grey arrow indicates the location of the $\Delta \mathrm{F} 508$ mutated residue.

\subsection{Binding Sites Prediction}

It is expected that conformational changes within NBD1 impact its molecular interaction with small molecules. Thus, cluster analysis was exploited to select from MDS trajectory the most representative $\triangle \mathrm{F} 508-\mathrm{NBD} 1$ conformation. Three clusters were obtained (with $51.5 \%, 25.6 \%$ and $9.1 \%$ of frames grouped with a distance $<2 \AA$ ). From the most populated one we extracted the most representative conformation that was then used to perform docking analysis with the ligands (Figure 3) using both blind and local approaches. As a result, a putative binding site and binding mode could be rationally inferred for each ligand. For aminoarylthiazole (AAT) derivatives, equi-populated clusters of solutions with similar energy and inverse orientation of the ligand were observed in each run (Supplementary Materials, Table S1, Figures S1-S3). 


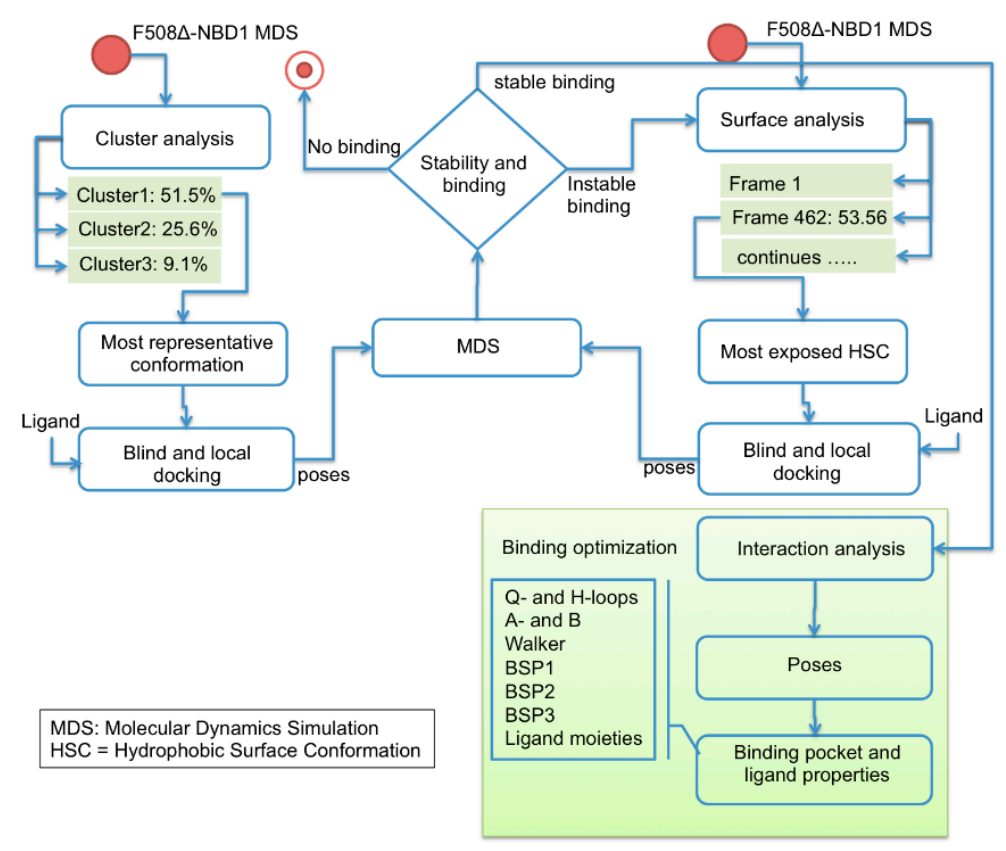

Figure 3. Workflow of the computational approach applied for binding site prediction and optimization.

Additional MDS were performed for each complex obtained from the docking calculations to optimize the orientation and pose of the ligand in the binding site and to highlight the ligand's moieties involved in its interaction with the NBD1 domain. In addition, the values of root mean square deviation (RMSD) between the starting and the terminal positions during MD simulations were calculated for each ligand to evaluate its stability inside the BSP. VX809 and the two AAT derivatives 4 and 6 retain their initial position consistently during MDS, thus remaining steadily anchored to $\triangle$ F508-NBD1 via the same interactions all along the trajectory (Figure 4).
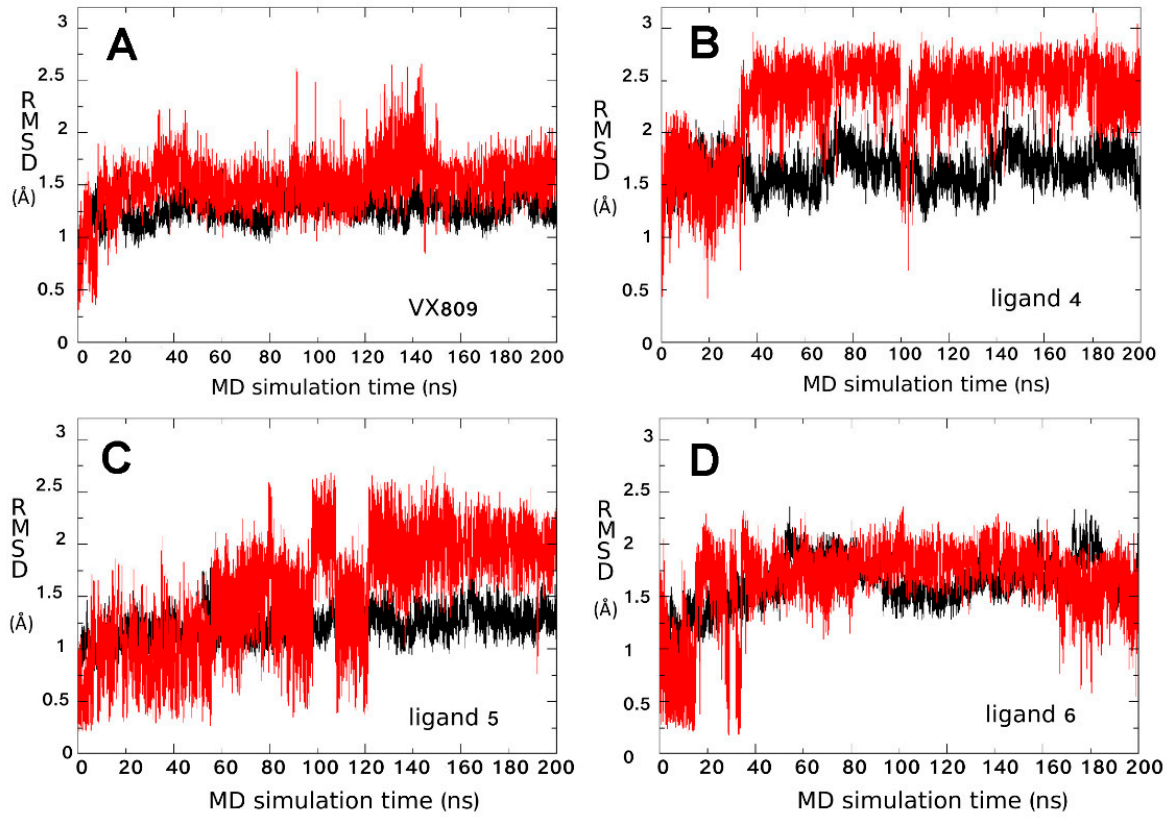

Figure 4. Analysis of putative binding mode stability. RMSD along simulation time for the $\triangle$ F508-NBD1/ligand complexes calculated with respect to the most representative conformation of the $\Delta$ F508-NBD1/ligand trajectory obtained from cluster analysis. The ligand and the $\Delta$ F508-NBD1 RMSD are depicted in red and in black, respectively. (A) VX809; (B) ligand 4; (C) ligand 5; (D) ligand 6. 
A highly unstable binding was instead observed during the simulation for AAT derivative 5 (Figure 4C and further investigated below), for the inactive AAT derivative 7 (included in the study to test the validity of the experimental protocol), for corrector 4a, (C4a) [11] and for VX770 (included in the study since they have been proposed to act by a mechanism of action independent from the NBD1 module) (data not shown). Accordingly, SPR analysis demonstrated that C4a and VX770 actually do not bind to $\triangle$ F508-NBD1 (Table 1 ).

Table 1. Affinity values $\left(K_{d}\right)$ of the interactions of CF putative drugs with $\Delta$ F508-NBD1 calculated by SPR analysis. $K_{d}$ values were derived by Scatchard's plot analysis of the equilibrium binding data. The results shown are the mean \pm standard deviation (S.D.) of two-three separate analyses. n.d.: not determinable.

\begin{tabular}{cc}
\hline Interaction & $\boldsymbol{K}_{\boldsymbol{d}}(\mu \mathbf{M})$ \\
\hline VX770/ $\Delta$ F508-NBD1 & n.d. \\
C4a/ $\Delta$ F508-NBD1 & n.d. \\
VX809/ $\Delta$ F508-NBD1 & $24.2 \pm 6.2$ \\
4/ $\Delta$ F508-NBD1 & $99.3 \pm 14.5$ \\
$5 / \Delta$ F508-NBD1 & $40.3 \pm 2.5$ \\
6/ $\Delta$ F508-NBD1 & $197.9 \pm 4.5$ \\
$7 / \Delta$ F508-NBD1 & n.d. \\
\hline
\end{tabular}

The binding instability of compound 5 observed during MDS (Figure 4C) was further investigated in order to predict the protein conformation able to give a stable ligand-protein complex. Analysis of the RMSD fluctuations as a function of time for the complex showed instability reflecting an unfolding of the $\mathrm{C} / \mathrm{N}$ terminal of the helix $9 \mathrm{X}$-ray structure.

A broader conformational freedom of $\triangle$ F508-NBD1 apo in respect to that of the WT NBD1 apo was observed, mainly due to the reorganization of the interactions network between residues and causing the exposure of a wider hydrophobic region [31]. On this basis, we searched for the best $\triangle$ F508-NBD1 conformation able to bind compound 5 in a stable way: by performing a hydrophobic surface analysis considering 1000 equidistant frames along the MDS of the $\triangle$ F508-NBD1 apo (one each 200 ps), we observed that the hydrophobic exposed surface spanned from 45 to $53 \AA^{2}$ (Figure 5A).
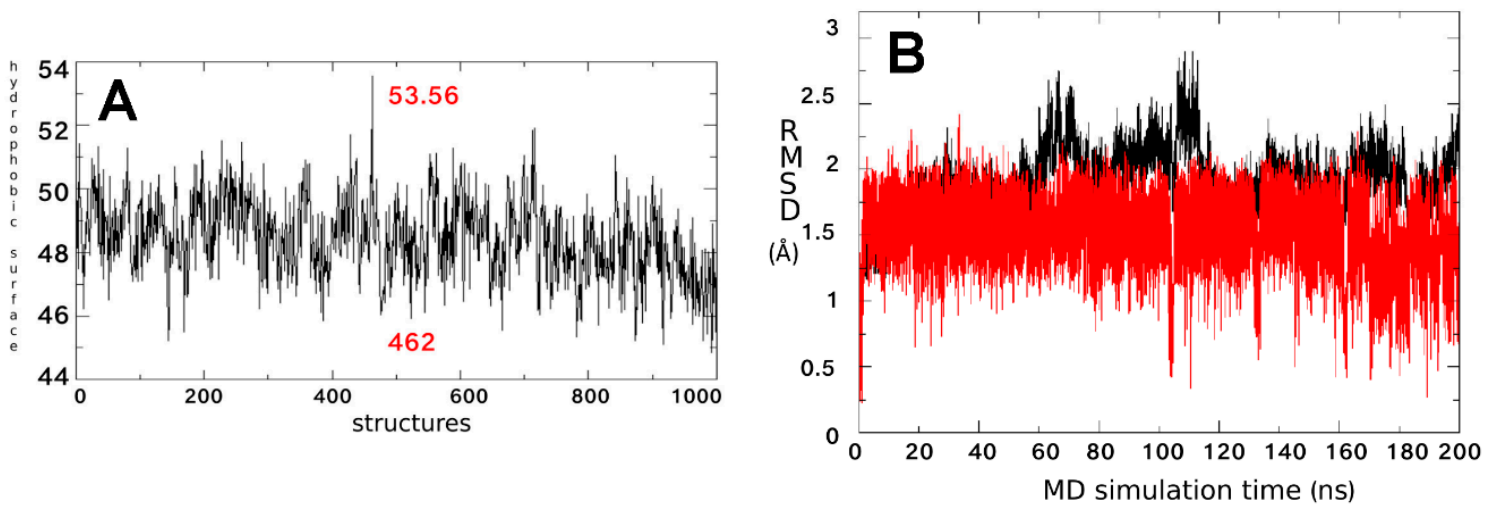

Figure 5. (A) Fluctuation of the hydrophobic surface area of $\triangle$ F508-NBD1 calculated considering 1000 equidistant structures along its dynamics (one each 200 ps). The frame number 462 with the most exposed hydrophobic surface used for compound 5 docking studies and 53.56, the value corresponding to its hydrophobic surface in $\AA^{2}$, are indicated in red; (B) RMSD of atomic loci along the MDS trajectory of the $\triangle$ F508-NBD1/compound 5 complex calculated in respect to the frame number 462 and plotted against simulation time. Black and red lines refer to $\triangle \mathrm{F} 508-\mathrm{NBD} 1$ backbone and ligands, respectively. All structure indices in $\mathrm{x}$-axis must be multiplied by 1000 to obtain the frame index. 
The frame with the most hydrophobic exposed surface was then taken in consideration as the potential protein conformation able to stably bind compound $\mathbf{5}$ and used to repeat docking studies (Figure 3, right side). In this way, a new binding site could be predicted where the ligand locates with high stability, at variance with what observed for the binding site previously predicted with the docking simulations based on the most representative conformation of $\triangle$ F508-NBD1 apo (Figure 4C).

\subsection{Ligand Interactions on $\triangle F 508-N B D 1$}

Ligand interactions information obtained from MDS predicted that the most effective ligand VX809 is anchored in the BSP1 region of $\triangle F 508-N B D 1$ by three H-bonds, the first between its amidic nitrogen and Y577, the second between its carbonyl group and E655 and the third between one of its catechol oxygens and V580 (Figure 6A and Supplementary Materials, Figures S4 and S6).
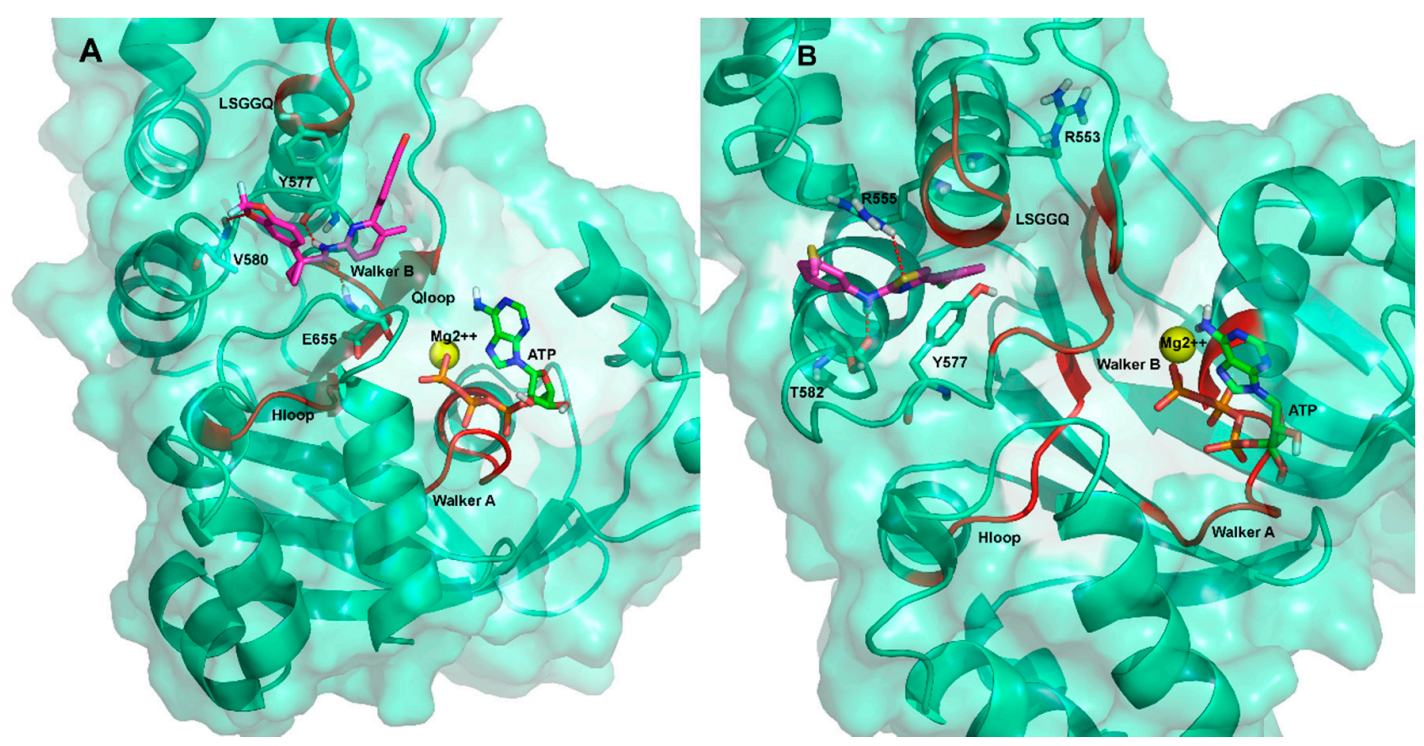

Figure 6. Predicted binding mode of VX809 (A) and compound 5 (B) with $\Delta$ F508-NBD1. The residues involved in the $\mathrm{H}$-bonds between the ligands and the mutated protein are shown as sticks in red dotted line. The protein is shown as ribbon. Conserved NBD signature sequences are shown as red ribbon.

Binding of compound 5 seems to occur in another NBD1 sub-pocket (BSP2) via a network of H-bonds: one between the sulphur atom of the AAT ring and R555, a very important residue of the RXR motif [33], another between the hydrogen of the aminic nitrogen of compound 5 and T582. Also, a $\pi-\pi$ interaction between the thiazolic ring of the AAT and Y577 seems to occur that further stabilizes the interaction (Figure 6B and Supplementary Materials, Figure S6).

Compounds 4 and 6 seem to bind to a further different sub-pocket (BSP3). Compound 4 making two H-bonds between the sulphur atom of its thiazole ring and R553 and between its amidic nitrogen and N416 (Figure 7A and Supplementary Materials, Figure S5). Concerning the compound $6 / \triangle$ F508-NBD1 interaction, cluster analysis on MDS trajectory predicted three equipopulated clusters with a $17 \%$ of occurrence each. This result is different from the other complexes analyzed, where occurrences of the most representative cluster are $46 \%, 38 \%$ and 33\% for VX809, compound 4 and compound 5, respectively. In all the clusters, a H-bond between compound 6 and S495 (a residue located in the Q-loop) can be predicted, while only in one cluster a transient H-bond between compound 6 and R555 seems to occur, due to alternative orientations of the side chain of the residue during the dynamics. Thus, at variance with VX809, all the other compounds studied seem to bind $\triangle F 508-N B D 1$ via residues belonging to the conserved NBD signature sequences, further supporting the hypothesis of different binding modes of the ligands to NBD1. Important to note, the binding modes here proposed for VX809 do not fully agree with some literature results proposing 
binding poses at the ICL4:NBD1 inter-domain interface and along strand S9 and strand S10 [10-13,15]. It must be pointed out however that all these studies were performed on RI-del human NBD1, without performing MDS to evaluate the stability complex.
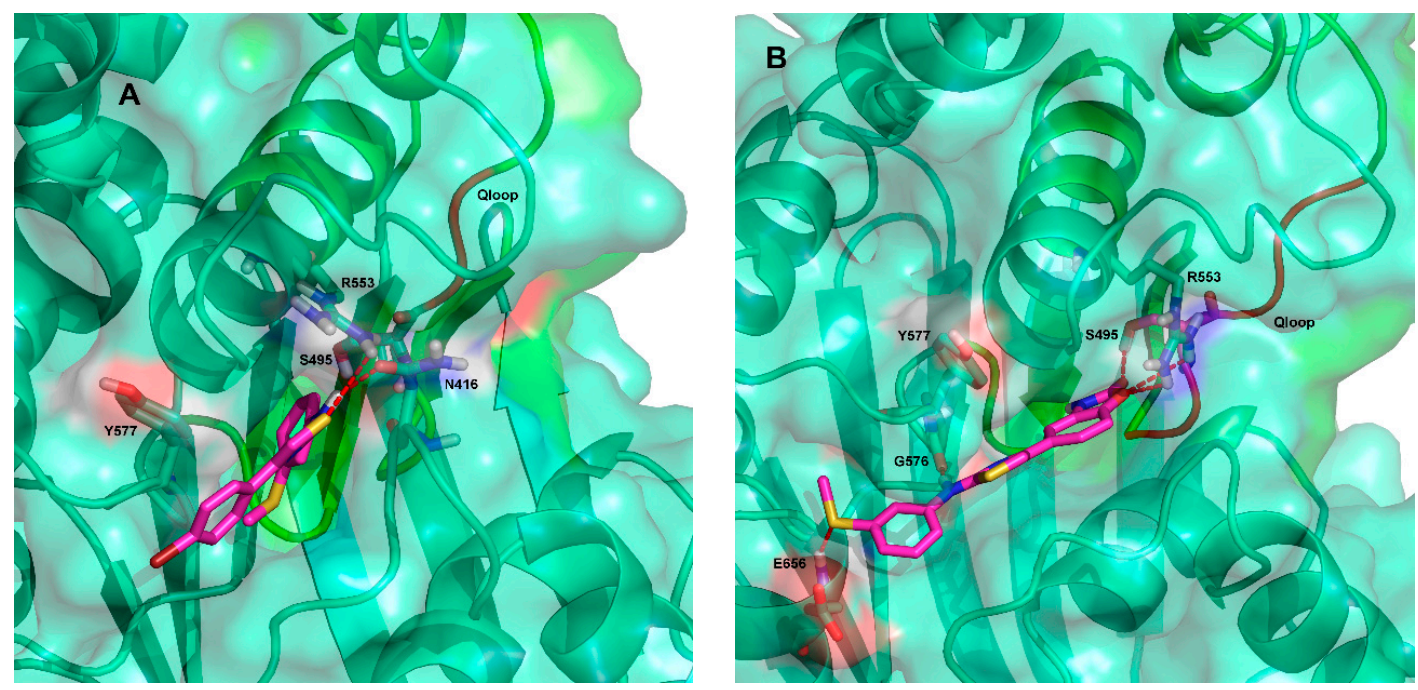

Figure 7. Predicted binding mode of compound 4 (A) and compound 6 (B) with $\triangle F 508-N B D 1$. The residues involved in $\mathrm{H}$-bonding interactions with the ligands are shown in sticks. The protein is shown as ribbon and Connolly surface. The binding region of K8 suggested by Odolczyk [34] is shown in green.

In addition, the RMSF profiles obtained from our VX809 binding pose, selected by docking and followed by MDS, show an attenuation of the fluctuation along almost all regions in $\triangle F 508-N B D 1$ when compared with the WT protein (Figure 8). This result is in agreement with literature data reporting reduced fluctuations induced by VX809 in 499-502 and 539-542 regions near ICL3 of $\Delta$ F508-NBD1 [35].

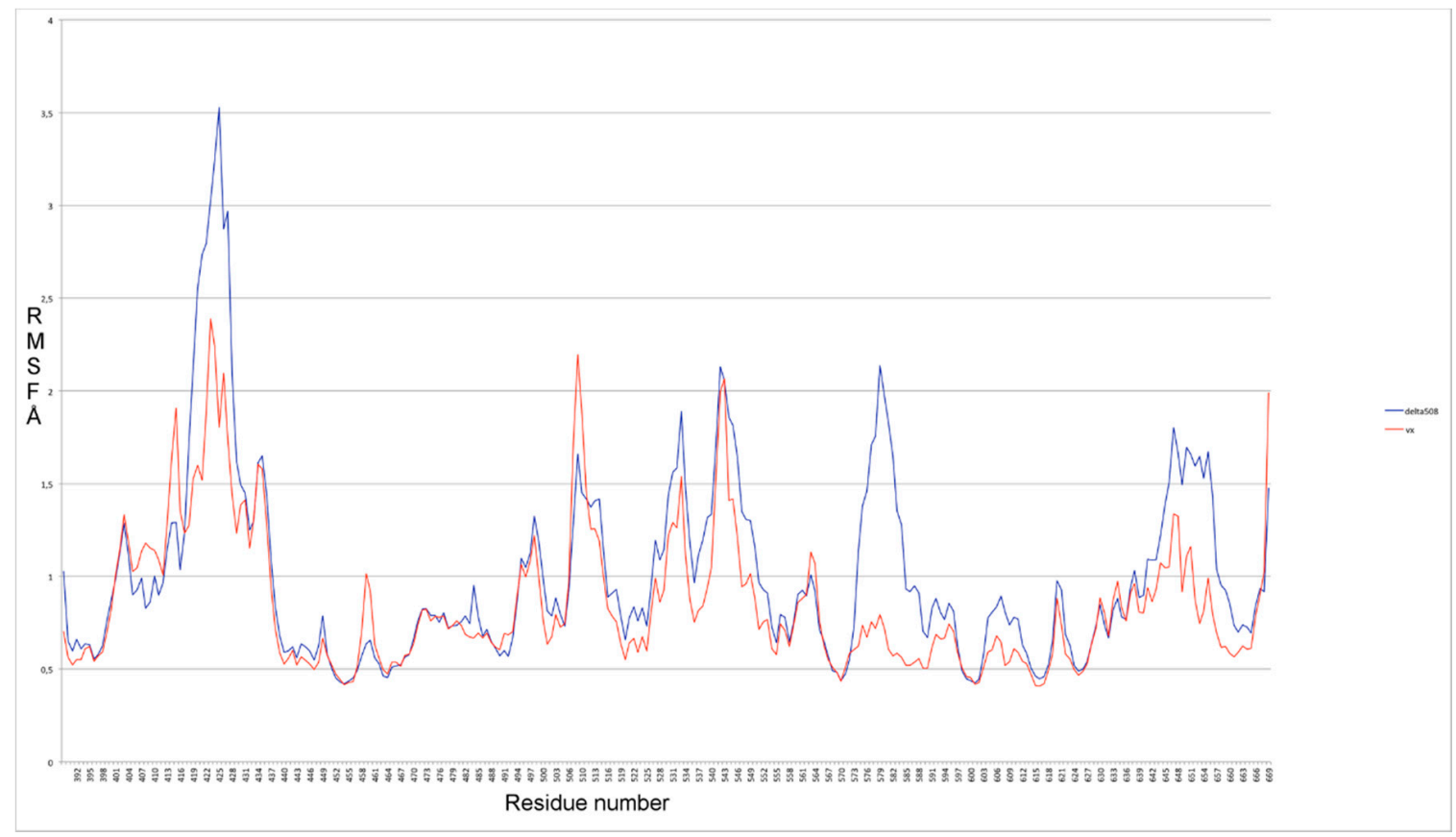

Figure 8. RMSF profiles obtained from VX809 binding pose in comparison with $\triangle$ F508-NBD1. 
Some CFTR-targeted drugs exert their action by impairing the binding of $\triangle \mathrm{F} 508$-NBD1 to K8, thus escaping the ubiquitin-proteasome machinery. This observation, together with the different positioning of the various AAT derivatives in the NBD1 module, prompted us to evaluate the capacity of the various compounds studied to interfere with the K8/ $\triangle F 508-N B D 1$ interaction. As a result, the binding pockets of VX809 and 5, as determined by MDS and docking (BSP1 and BSP2, respectively), turned out to be distinct from the binding site of K8 already reported by Odolczyk [34]. The binding modes of compounds $\mathbf{4}$ and $\mathbf{6}$ indicated instead that they share part of their binding pocket with the K8 anchoring cleft. In details, the aromatic ring of compound 4 bringing the methylsulphur moiety seems to be partially inserted into the anchoring cleft of K8 (Figure 7A), while the 2-oxazolidinone moiety of compound $\mathbf{6}$ appears to be completely inserted into an inner portion of the K8 anchoring cleft, with the remaining part of the molecule situated in the adjacent sub-pocket, making two H-bonds between its aminic nitrogen and G756 and between the sulphur atom of its methylsulphur moiety and E656 (Figure 7B and Supplementary Materials, Figure S7).

\subsection{Interaction Analysis by Surface Plasmon Resonance}

SPR analyses were finally performed to validate the in silico predictions that, as already stated above, is the main aim of this work. In a first series of experiments, to check the correct immobilization and binding availability of $\triangle \mathrm{F} 508$-NBD1 onto the sensor chip, we performed binding analyses with $\mathrm{K} 8$ in those same experimental conditions used to analyze the binding of the putative $\mathrm{CF}$ drugs (see Materials and Methods). As shown in Figure 9A, when tested at $600 \mathrm{nM}, \mathrm{K} 8$ specifically binds to immobilized $\triangle$ F508-NBD1 but not to BSA surface (here used as a negative control). When increasing concentrations of $\mathrm{K} 8$ were injected onto the $\triangle \mathrm{F} 508$-NBD1 surface, the blank subtracted sensorgrams overlay shown in Figure 9B was obtained: K8/ $\triangle$ F508-NBD1 interaction occurs in a saturable and very stable way, being $\mathrm{K} 8$ detachment from the sensor chip after the end of the injection very slow.

Once validated the experimental model, the direct interaction of the putative CF drugs with $\triangle$ F508-NBD1 was investigated. In agreement with computational predictions, VX770, C4a and compound 7 do not bind to $\triangle \mathrm{F} 508-\mathrm{NBD} 1$ (Table 1). The other compounds bind to $\triangle \mathrm{F} 508-\mathrm{NBD} 1$ but, at variance with $\mathrm{K} 8$, their interaction is characterized by a very quick detachment from the sensor chip after the end of the injection (Figure 9A-D). For compounds 4 and $\mathbf{5}$ in particular, their premature detachment from surface-immobilized $\triangle \mathrm{F} 508$-NBD1 can be appreciated even during the association phase, causing an anomalous shaped sensorgram. This may be explained by the limited solubility of small chemical compounds and their tendency to aggregate during the SPR analysis also in the presence of DMSO [36].

Blank subtracted values of RU bound at equilibrium obtained from injections of increasing concentrations of the various putative CF drugs on $\triangle F 508-N B D 1$ were then used to build the curves reported in Figure 9E-H: VX809, compounds $\mathbf{5}$ and $\mathbf{4}$ bind to $\triangle$ F508-NBD1 in a dose-dependent and saturable manner, with $K_{d}$ values ranging between $24.2 \pm 6.2$ and $99.3 \pm 14.5 \mu \mathrm{M}$. At the concentrations tested, compound 6 shows no saturable binding, with a presumed $K_{d}$ value equal to $197.9 \pm 4.5 \mu \mathrm{M}$ (Table 1).

In silico studies predicted VX809 and compound 5 to locate in two distinct regions of $\triangle$ F508-NBD1 (BSP1 and 2, respectively) and compounds 4 and 6 to locate in region BSP3 proposed to be also involved in the binding to $\mathrm{K} 8$ [37]. These observations prompted us to evaluate the capacity of the various compounds to affect the binding of $\mathrm{K} 8$ to $\triangle \mathrm{F} 508-\mathrm{NBD} 1$. In a first series of experiments, exploiting the slow dissociation rate of the $\mathrm{K} 8 / \triangle \mathrm{F} 508-\mathrm{NBD} 1$ interaction, a saturating amount of the chaperone was injected over the $\triangle \mathrm{F} 508$-NBD1 surface and allowed to reach an equilibrium binding. Subsequently, the putative drugs were injected. 

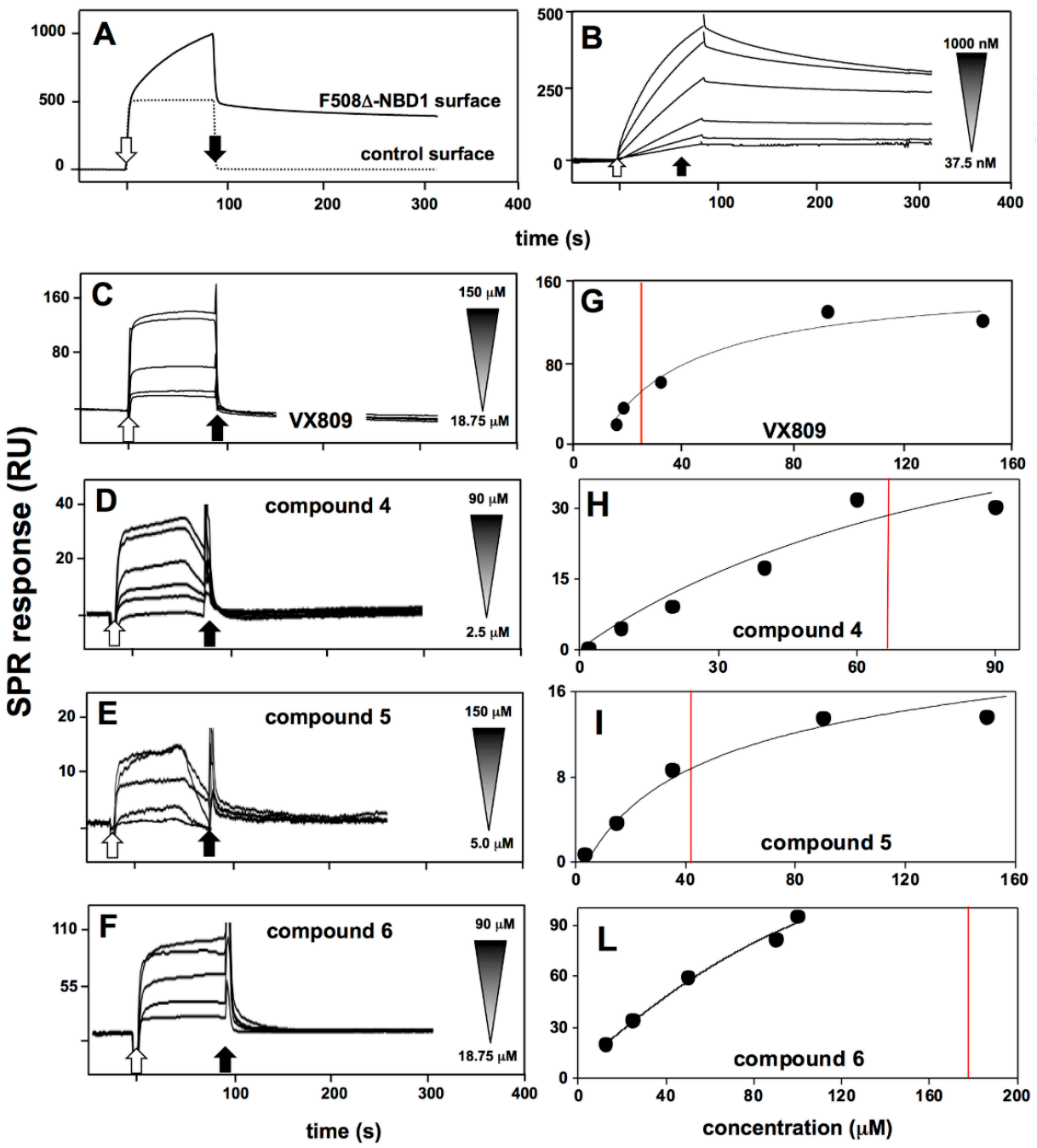

Figure 9. SPR analysis of the interaction of $\triangle F 508-N B D 1$ with $K 8$ or with the putative CF drugs. (A) Sensorgrams showing the binding of $\mathrm{K} 8(600 \mathrm{nM})$ to a $\triangle$ F508-NBD1- or to a control BSA-coated surface; (B) Blank-subtracted sensorgrams overlay showing the binding of increasing concentrations of $\mathrm{K} 8(1000,600,300,150,75,37.5 \mathrm{nM})$ to the $\Delta$ F508-NBD1-coated sensor chip; (C-F) Representative blank-subtracted sensorgrams overlays showing the binding of increasing concentrations of the indicated compounds to the $\triangle$ F508-NBD1-coated sensor chip. In all the panels, response (in RU) was recorded as a function of time. White and black arrows mark the beginning and the end of the injection of K8 or of the compounds; (G-L) Steady-state analysis obtained by fitting the proper form of Scatchard's equation for the plot of the bound RU at equilibrium versus the ligand concentration in solution for the indicated compounds injected onto the sensor chip-immobilized $\triangle$ F508-NBD1. Red lines indicate the calculated $(\mathbf{G}, \mathbf{H})$ or presumed $(\mathbf{L}) K_{d}$ values.

As shown in Figure 10A, injection of VX809 or of compound 5 on the K8-saturated $\triangle$ F508-NBD1 surface results in an increase of bound $\mathrm{RU}$, indicating that these two compounds retain the capacity to bind to $\triangle \mathrm{F} 508$-NBD1 also when the protein is already engaged by K8. Relevant to this interpretation, none of the compounds studied is able to bind to K8 (Figure 10B). The inability of compounds 4 and 6 to alter the binding of $\mathrm{K} 8$ to $\triangle \mathrm{F} 508-\mathrm{NBD} 1$ (Figure $10 \mathrm{~A}$ ) may be due to the fact that the $\Delta \mathrm{F} 508-\mathrm{NBD} 1 / \mathrm{K} 8$ interaction is too stable to be disrupted by the subsequent injection of the two compounds that indeed turned out to be the two weakest $\triangle$ F508-NBD1 binders (Table 1 ). In any case, the capacity of both the AATs and $\mathrm{K} 8$ to bind to immobilized $\triangle \mathrm{F} 508-\mathrm{NBD} 1$ makes this experimental model inappropriate to study possible competitive effects. To overcome this limit, we reversed the SPR model by immobilizing 
$\mathrm{K} 8$ and evaluating its capacity to bind $\triangle \mathrm{F} 508-\mathrm{NBD} 1$ (injected at $600 \mathrm{nM}$ ) in the presence of the compounds at a dose $(500 \mu \mathrm{M})$ compatible with the highest concentration of DMSO $(5 \%)$ that does not hamper the K8/ $\triangle \mathrm{F} 508-\mathrm{NBD} 1$ interaction (data not shown). As already mentioned, none of the compounds interacts with immobilized K8 in these experimental conditions (Figure 10B). As shown in Figure 10C, when injected alone, $\triangle$ F508-NBD1 binds to immobilized-K8 (35 RU at equilibrium). The injection of $\triangle \mathrm{F} 508-\mathrm{NBD} 1$ in the presence of VX809 or compound 5 results in an interaction that is quantitatively higher (68 and $45 \mathrm{RU}$ at equilibrium, respectively), confirming the results obtained in the previous SPR assay. On the contrary, in the same experimental conditions, compound 4 exerts a $50 \%$ inhibition of the $\triangle \mathrm{F} 508$-NBD1/K8 interaction, indicating that it competes with immobilized K8 for the binding to $\triangle$ F508-NBD1 while compound 6 resulted again ineffective, possibly because of its extremely low affinity interaction with $\triangle \mathrm{F} 508$-NBD1 (Table 1). In conclusion, also when dealing with the effect of the drugs on $\triangle \mathrm{F} 508-\mathrm{NBD} 1 / \mathrm{K} 8$ interaction, computational predictions are validated by experimental SPR analysis.

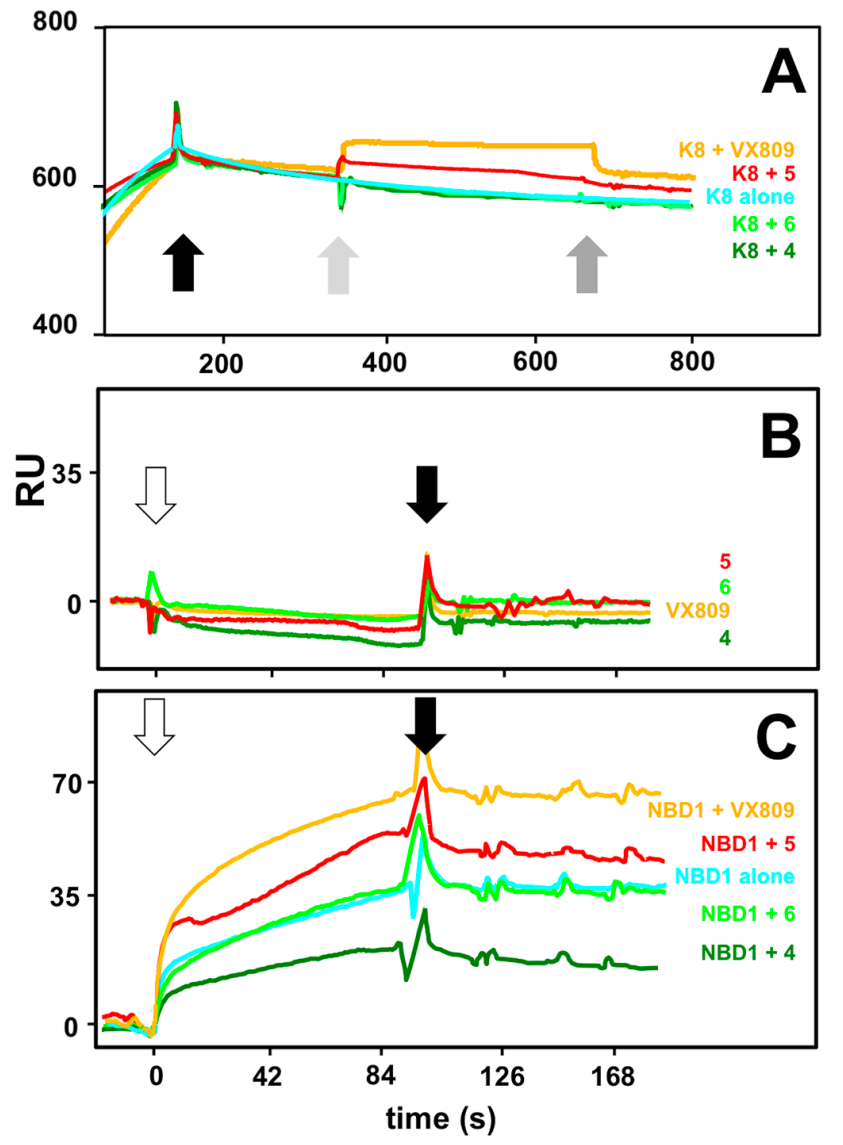

Figure 10. Effect of the putative CF drugs on the K8/ $\triangle F 508-N B D 1$ interaction. (A) Representative sensorgrams showing the injection of the various compounds (at $150 \mu \mathrm{M}$ ) onto $\Delta \mathrm{F} 508$-NBD1-coated sensor chip saturated with K8. The black arrow marks the end of K8 injection. Light and dark grey arrows mark instead the beginning and the end of the subsequent injection of the various compounds on the K8-saturated surface; (B) Representative sensorgrams showing the injection of the various compounds $(500 \mu \mathrm{M})$ onto K8-coated sensor chip. White and black arrows mark the beginning and the end of the injection of the compounds, respectively; $(C)$ Representative sensorgrams showing the injection of $\triangle$ F508-NBD1 (600 nM) onto K8-coated sensor chip in the absence or in the presence of the indicated compounds $(500 \mu \mathrm{M})$. White and black arrows mark the beginning and the end of the injection of the various mixtures, respectively. In all the panels, the response (in RU) was recorded as a function of time. The sensorgrams shown are representative of other two that gave similar results. 
A last relevant aspect is the statistically significant correlation that exists, for each compound, between the values of maximal RU bound to sensor chip-immobilized $\triangle F 508-\mathrm{NBD} 1$ at equilibrium and the different time availability of the $\triangle$ F508-NBD1 pockets as calculated from the docking poses simulation along the MD of the protein in apo form (Supplementary Materials, Figure S4). VX809 binds to the highest number of available $\triangle \mathrm{F} 508$-NBD1 conformations $(48 \%)$ and, accordingly, it shows also the highest binding capacity when tested in SPR (Supplementary Materials, Table S2). The AAT derivatives behave differently, with compounds 5 and 4 able to bind only $15 \%$ of the available conformations and compound $\mathbf{6}$ showing an intermediate capacity $(22 \%)$.

Again, these values quantitatively correlate to the values of maximal RU bound at equilibrium (Supplementary Materials, Table S2). The disclosed correlation between these latter values and the different time availability of the $\triangle \mathrm{F} 508-\mathrm{NBD} 1$ pockets suggests that $\triangle \mathrm{F} 508-\mathrm{NBD} 1$ retains its capacity to present different conformations also when immobilized to the sensor chip. As a result, the different time availability of its distinct binding pockets reflects in a different binding capacity that could be not directly related to the measured binding affinity. The take-home message is that both pocket availability (as predicted by computational studies) and the values of maximal binding at equilibrium (as calculated by SPR analysis) should be taken into account when evaluating the efficacy of a potential lead compound, further underlying the validity of the double approach here proposed.

\section{Discussion}

In this work, by means of extensive MDS simulation and docking, we have studied the interaction of $\triangle$ F508-NBD1 with a series of AAT derivatives and VX809, selected as template. The results of these studies allowed the prediction of putative binding modes with statistics of occurrence that finally turned out to be in agreement with actual SPR binding analysis, linking solidly these two complementary techniques. This in-tandem approach, here applied to the murine NBD1 domain, can contribute to unravel the complex puzzle concerning the mechanism of action of small molecules in CFTR-targeted therapy, an issue that is commonly acknowledged as not fully understood, due mainly to the still present limits of the experimental structures of human NBD1 and complete CFTR (see Introduction).

The computational investigations on $\triangle \mathrm{F} 508$-NBD1 here performed provided novel insights regarding the binding mode of known and novel potential CF drugs: the well-known VX809 was predicted to strongly anchor to the central cleft of NBD1 via three H-bonds. Accordingly, when compared to the ATT derivatives in SPR analysis, it shows the highest binding affinity for $\triangle$ F508-NBD1 (Table 1). In respect to VX809, the three AAT derivatives 4,5 and $\mathbf{6}$ seem not to share the same binding site, even if endowed of the same chemical scaffold. In details, compound 6 appears to occupy a sub-region inside the main pocket, orienting its carbonyl group towards S495 in the Q-loop. Compound 4 presents a slightly different predicted binding since in its scaffold is present a $p$-bromophenyl moiety that is less polar than compound $\mathbf{6}$ and whose carbonyl group causes the molecule to accommodate in the same sub-pocket but in a different mode, flanking the RXR motif. Interestingly, modelling and mutagenesis studies on NBD1/NBD2 dimers showed that activator compounds strongly interact with the NBD1 domain in a site that includes part of the Q-loop, of the LSGGQ signature, of switch-II of NBD1 plus the Q-loop of NBD2 [38]. These computational findings support the evidence that compound $\mathbf{6}$ acts as a potentiator [26]. Furthermore, NMR spectra of various NBD1 mutants support the evidence that deletion of Phe-508 affects Q-loop conformation inhibiting dimerization, possibly explaining the mechanism by which the mutation impairs the opening of the channel [38] and rationally supporting the selection of $\triangle \mathrm{F} 508-\mathrm{NBD} 1$ as the primary target for designing new activators.

Compound 5 is predicted to bind to BSP2 inside the main pocket, closer to the LSSGQ conserved residues and specifically interacting with R555 of the RXR motif, a conserved portion of NBD1 already reported to be important for CFTR correct folding and stability [39]. Thus, a possible mechanism of action of compound 5 can be its capacity to prevent the abnormal accessibility of RXR in $\triangle$ F508-NBD1, 
thus preventing mistrafficking of the mutated protein. Also, the predicted different binding modes of compound 5 and VX809 are in agreement with the observation that the two compounds show a synergistic effect in vitro [26].

The predicted distinct binding modes of the AAT derivatives correlate to their different scaffold decorations and support their double biological activity [23]. In fact, according to our calculations, the interaction of compound $\mathbf{6}$ with S495 residue in the Q-loop may promote the dimerization of NBD1 with NBD2 and thus the following channel opening (potentiator activity). On the other hand, the predicted H-bonds of compounds 5 and 4 with the arginine residues of the RXR motif may alter the interaction between $\triangle \mathrm{F} 508-\mathrm{NBD} 1$ and the proteostasis components (CFTR interactome) allowing the protein to reach the plasma membrane (corrector activity).

Finally, it is interesting to note that the binding modes we have inferred by MDS and docking suggest that compounds 4 and $\mathbf{6}$, but not VX809 and compound 5, partially insert in the proposed K8 binding site of $\triangle \mathrm{F} 508-\mathrm{NBD} 1$, each one in a specific way.

These predicted binding modes were validated by SPR analysis that showed that compound 4 displaces K8 from its binding to $\triangle$ F508-NBD1, while VX809 and compound 5 can bind the protein simultaneously to $\mathrm{K} 8$. This also suggests the possibility that the well demonstrated rescuing capacity of VX809 in vivo [23,26] is K8-independent, being possibly mediated though the disruption of the interaction of $\triangle \mathrm{F} 508-\mathrm{NBD} 1$ with chaperone(s) other than K8. This calls for further studies aimed at evaluating the effect of VX809 (as well as of other compounds) on chaperones different from K8 (i.e., Hsp70/Hsc70, Hsp90, CHIP and BAG-2) [3]. On the other hand, in silico predictions and SPR analysis point to compound 4 as a very interesting lead compound being able to displace $50 \%$ of $\mathrm{K} 8$ in its binding to $\triangle \mathrm{F} 508$-NBD1. In effect, as already reported by Colas et al. [4], the disruption of $\mathrm{K} 8 / \triangle \mathrm{F} 508-\mathrm{NBD} 1$ interaction leads to the functional correction of the mutated protein. Further studies are required to fully clarify these points since the specific residues of $\triangle \mathrm{F} 508-\mathrm{NBD} 1$ that directly interact with K8 (as well as with other chaperones) still need to be experimentally defined.

In conclusion, the in-tandem approach made up of in silico studies and SPR analysis points to different binding modes (and different binding affinity) for VX809 and the various AATs that, in turn, indicate two potential rescue mechanisms: the first consisting in the capacity of the compound (i.e., VX809 and compound 5) to stabilize the conformational variability of NBD1, the second consisting instead in the capacity of the compound (i.e., compound $\mathbf{4}$ and possibly compound $\mathbf{6}$ ) to interfere with the binding of $\mathrm{K} 8$ to $\triangle \mathrm{F} 508-\mathrm{NBD} 1$.

Finally, it is interesting to note that the SPR-generated values of RU bound at equilibrium to $\triangle F 508-N B D 1$ and the docking poses of $\triangle$ F508-NBD1-ligand evaluated by inverse docking technique (Supplementary Materials, Table S2) correlate in a statistically significant way (Supplementary Materials, Figure S4), suggesting that the former parameter depends on the actual availability of the binding pockets that are variably exposed during the structural fluctuation of $\Delta$ F508-NBD1. In turn, this indicates that, once immobilized to a surface, the mutated protein maintains (at least in part) its capacity to move in the space, assuming those different conformations assessed by computational analysis.

The results of this work open up to further researches with multiple purposes: (a) to validate the putative binding modes of the compounds with $\triangle$ F508-NBD1 mutagenesis in those amino acids predicted to be involved in the interactions; (b) to correlate the various disclosed binding modes to different recovery effects of the compounds on $\triangle \mathrm{F} 508$-CFTR by means of in vitro experiments; (c) to study the effect of selected compounds on the interaction of $\triangle$ F508-NBD1 with chaperones other than $\mathrm{K} 8$; (d) to speed up the design and synthesis of new and more efficient AAT derivatives, able to correct the $\triangle$ F508-NBD1 defect; (e) to better understand the molecular mechanisms of interactions and action of the new compounds, useful for a more exhaustive knowledge of CFTR disease. 


\section{Materials and Methods}

\subsection{Computational Studies}

\subsubsection{Ligand Dataset}

The ligand dataset has been chosen including three well known compounds provided by L.J.V. Galietta and here used as references: VX809 (Lumacaftor, one of the most effective correctors [24]); VX770 (Ivacaftor, a potentiator that does not bind to NBD1 [22] and that has been recently approved for CF therapy by both FDA and EMEA); C4a that, as VX770, does not bind to NBD1 [11,25]. Four new AAT derivatives, already synthesized by some of us, were also included: compounds 4, 5, 6 and the inactive compound 7 [23,26] (Figure 1). All compounds were built, parameterized (Gasteiger-Hückel method) and minimized with OMEGA 2.5.1.4: (OpenEye Scientific Software, Santa Fe, NM, USA, www.eyesopen.com) using MMFF94 force field. Regarding VX809 (characterized by a carboxylic moiety), accurate pKa predictions performed with ChemSpider (a free web tool from the Royal Society of Chemistry that can be found at: http:/ / www.chemspider.com) let us to reasonably suppose that it maintains its anionic form at physiological $\mathrm{pH}$, and calculations were performed accordingly.

\subsubsection{Model Refinement}

Since the WT and $\triangle$ F508-NBD1 proteins available for our SPR analysis were the murine forms obtained from CFTR folding consortium (see below), we performed all computational studies using the murine crystallographic structures.

WT- and $\triangle$ F508-NBD1 models (spanning residues 389-673) were based on the crystal structure of NBD1 from mouse CFTR, 1R0Z [40] and 3SI7 [41]. The phosphorylation on residues Ser422, Ser659, Ser660, and Ser670 were removed. Both structures presented an ATP-Mg ${ }^{2+}$ complex. The gap of six residues spanning positions 414-419 present on the WT NBD1 was modelled ab initio with loop refinement algorithm of Modeller [42], a tool relying on a scoring function and on an optimization schedule suitable for loop modelling. In details, hundreds of loop models were generated and their potential energy was evaluated with the DOPE scoring function. The lowest energy loop model was then chosen and the resulting WT model was used as a template to bridge the gap in the mutant domain (spanning residues 414-428).

\subsubsection{MDS Simulations}

MD simulations were carried out using Amber 12 Molecular Dynamics Package. The compounds and the proteins were parameterized by means of the empirical charge model AM1-BCC (using the Antechamber module) and of the AMBER99SB-ILDN force field, respectively. The NBD1 module of CFTR was capped with an acetyl group on its $\mathrm{N}$-terminus and with a $N$-methylamide on its C-terminus. The complex was solvated in a truncated octahedron box ( $8 \AA$ distance between protein and box wall) using the explicit TIP3P water model. $\mathrm{Na}^{+}$ions were added to neutralize the whole system. Minimization of the system was performed by several stages of steepest descent and conjugate gradient to remove bad contacts and release restraints.

Using NVT conditions the system was weakly coupled to a temperature bath of $300 \mathrm{~K}$ under control with the Langevin algorithm that uses a simple Leapfrog integrator to propagate the dynamics. Next, in NPT conditions, the Berendsen pressure coupling of $1 \mathrm{~atm}$ with a collision frequency of $2 \mathrm{ps}^{-1}$ was applied. After equilibration, MDS trajectories were carried out for $200 \mathrm{~ns}$ with an integration step of $2 \mathrm{fs}$. All the bonds involving hydrogen atoms were constrained by the SHAKE algorithm. The non-bonded cut-off distance was set to $8 \AA$ and long range electrostatic interactions were calculated using Particle Mesh Ewald (PME) method. 


\subsubsection{Docking Studies, Ligand-Protein Docking}

The most representative conformations were obtained from the most populated cluster of the $\triangle \mathrm{F} 508-\mathrm{NBD} 1 \mathrm{MDS}$ using the backbone atoms to compute the distance between each pair of frames (Figure 3, left side). A sampling of 10,000 frames along the trajectory was used for cluster analysis. This conformation was used to perform ligand-protein docking calculations. We checked that the docking poses were correctly inside the docking binding sub-pockets in the putative binding pocket predicted by CASTp [43]. Autodock Tools [44] was used to prepare proteins and ligands for the docking computations and to analyze the results. Polar hydrogen atoms were added to proteins and ligands and non-polar hydrogen atoms were merged. Finally, Gasteiger charges were added to all atoms. Protein coordinates were set to be rigid while ligand bonds were set to be rotatable. Blind (no specific binding pocket is searched) and local (docking targeting a specific binding pocket) protocols were performed to predict the binding sites for each ligand of the dataset. In particular, in blind docking calculations, a grid box encompassing the entire NBD1 structure was generated to take in consideration all the possible NBD1 binding sites. Calculations were carried out using Auto-Dock Vina [45]. The exhaustiveness of the global search was set to 64 . The complex models with the lowest energy (based on an evaluation of AutoDock Vina's empirical scoring function) were selected.

A local docking set of experiments was performed to predict the binding of each ligand of the dataset and to optimize the ligand-protein interactions. Both Autodock Vina and AutoDock4 were used for local docking and finally only the latter was used due to ambiguities (same energy and different pose) in the choice of the best pose in Autodock Vina results. AutoDock4 was used with a grid box centered on the binding regions predicted in the blind docking calculations. The affinity maps for all the atom types present, as well as an electrostatic map, were computed with a grid spacing of $0.375 \AA$. The search was carried out with the Lamarckian Genetic Algorithm, with the number of generations, energy evaluations and docking runs set to 27,000, 2,500,000, and 100 respectively [44]. Considering only the most populated clusters, we retained a set of two or three clusters with similar energy and numerosity. For each cluster we selected the pose with the lowest energy and used the corresponding complex to run an MD simulation for $200 \mathrm{~ns}$ using the PME method. In this way we filtered out the dynamically unstable poses retaining only those that were stable.

This procedure allowed us to predict a stable docked pose for VX809, compounds 4 and $\mathbf{6}$ but not for compound $\mathbf{5}$ for which we used another approach: hydrophobic surface analysis performed on 1000 frames along the MDS trajectory of $\triangle F 508-N B D 1$ allowed the prediction of the frame with the most exposed hydrophobic surface (Figure 3, right side). Thereafter, the procedure was identical to the one described above (blind docking, MDS and stability analysis).

\subsubsection{Docking Studies, Inverse Docking Technique to Calculate Binding Propensity}

In order to try to explain the experimental RU values obtained by SPR analysis in relation to the pocket availability at equilibrium of the $\triangle \mathrm{F} 508-\mathrm{NBD} 1$, we used a ligand-protein inverse docking technique to obtain a measure of how many $\triangle \mathrm{F} 508-\mathrm{NBD} 1$ structural conformations along the MDS are able to bound to their respective ligands (Figure 11).

We implemented a protein pocket dataset that includes the three pockets (BSP1, BSP2, BSP3) obtained from the workflow illustrated in Figure 3, and performed docking analysis of each pocket against a selection of 1000 time-equidistant frames along the MDS of the $\triangle$ F508-NBD1 (one each $200 \mathrm{ps}$ ). The computations were carried out using local docking with Auto-Dock Vina. For each ligand/ $\triangle F 508-N B D 1$ docking simulation, the lowest energy complex was identified and for each ligand, all docked poses over the 1000 frames were grouped by similar ligand-pocket distance between the docking pose and the reference pose, the latter obtained considering the most frequent cluster of the dynamic of the complex. The results were then compared to the mean value of the binding energy of the group, allowing the evaluation of the number of $\triangle F 508-N B D 1$ conformations able to bind to each compound (Table S2). 


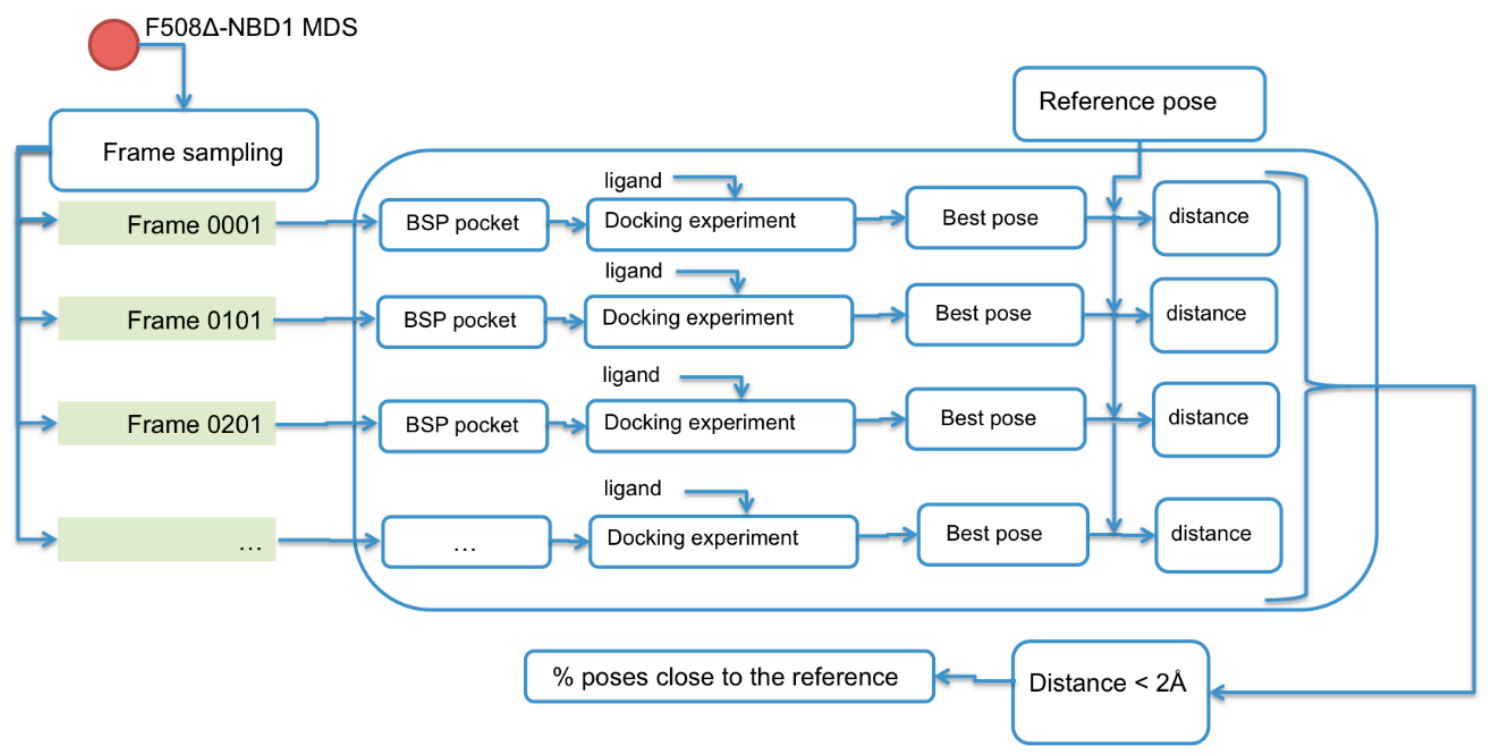

Figure 11. Workflow of the applied ligand-protein inverse docking technique.

\subsubsection{Trajectory Analysis}

MDS trajectories were analysed using the root mean square deviation (RMSD) and the root mean square fluctuation (RMSF) techniques plus the hydrophobic surface (HS). RMSD was used as a measure of the stability of the unbound proteins compared to the crystallographic structures and as a measure of the ligand complex along the dynamics compared to its starting pose obtained from docking. RMSF was used to determine the fluctuating regions in $\triangle F 508-N B D 1$, highlighting the differences in flexibility in respect to the WT form of the protein.

HS area was computed with the per-residue Shrake-Rupley method [46] using 1200 probes with a $1.4 \AA$ radius to evaluate the $\triangle \mathrm{F} 508-\mathrm{NBD} 1$ hydrophobic exposure area during the simulations.

\subsection{Reagents and Recombinant Proteins}

Murine WT NBD1 and $\triangle$ F508-NBD1 were obtained from CFTR Folding Consortium [37]. K8 was purchased by Pro-Spec-Tany TechnoGene Ltd. (Ness Ziona, Israel). The reagents 1-ethyl-3-(3-diaminopropyl)carbodiimide hydrochloride (EDC) and N-hydroxysuccinimide (NHS) were purchased from GE Healthcare (Milwaukee, WI, USA).

\subsection{Surface Plasmon Resonance (SPR) Binding Assay}

SPR measurements were performed on a BIAcore X-100 instrument (GE-Healthcare, Milwaukee, WI, USA), using research-grade CM5 carboxyl-methyl-dextran-coated sensor chips (GE-Healthcare). The murine $\triangle F 508-N B D 1$ was resuspended at $20 \mu \mathrm{g} / \mathrm{mL}$ in $10 \mathrm{mM}$ sodium acetate $\mathrm{pH} 4.0$ and allowed to react with the first flow cell of a CM5 sensor chip pre-activated with $50 \mu \mathrm{L}$ of $0.2 \mathrm{M}$ EDC and $0.05 \mathrm{M}$ $\mathrm{NHS}$ and deactivated by injection of $\mathrm{HCl}$-ethanolamine. This procedure led to the immobilization of $11,000 \mathrm{RU}$, equal to approximately $400 \mathrm{fmol} / \mathrm{mm}^{2}$ of protein. Similar results were obtained for the injection of BSA $(20 \mu \mathrm{g} / \mathrm{mL})$ onto the second flow cell of each sensor chip, used as a negative control and for blank subtraction. The appropriate immobilization of $\triangle \mathrm{F} 508-\mathrm{NBD} 1$ was checked by injecting increasing concentrations of K8 in PBS, $0.05 \%$ surfactant P20 and 5\% DMSO, pH 7.4 over the sensor chip for $90 \mathrm{~s}$ at $10 \mu \mathrm{L} / \mathrm{min}, 25^{\circ} \mathrm{C}$ and then washed until dissociation was observed. The inclusion of $5 \%$ DMSO in the running buffer (not necessary for protein-protein interaction analysis) and the short association time were chosen to validate $\triangle F 508$-NBD1 binding availability in the experimental conditions used for the subsequent analysis of the compounds (see below). 


\subsubsection{Direct Binding Analysis}

For the evaluation of their capacity to directly bind to $\triangle \mathrm{F} 508-\mathrm{NBD} 1$, increasing concentrations of the various compounds of the data-set were resuspended in PBS, $0.05 \%$ surfactant $\mathrm{P} 20$ and $5 \%$ DMSO, $\mathrm{pH} 7.4$, injected over $\Delta \mathrm{F} 508-\mathrm{NBD} 1$ or BSA surfaces for $90 \mathrm{~s}$ at $10 \mu \mathrm{L} / \mathrm{min}, 25^{\circ} \mathrm{C}$ and then washed until dissociation was observed. Steady state analysis were performed by fitting the proper form of Scatchard's equation for the plot of the bound RU at equilibrium versus the compound concentration in solution. When saturation binding was reached, $K_{d}$ values were calculated. In the case no saturation could be reached due the limited solubility of the compound under test, the software provided a $K_{d}$ value expected on the bases of the curve's trend.

In a further series of experiments, a saturating amount of $\mathrm{K} 8(1 \mu \mathrm{M})$ was injected on the $\triangle F 508-N B D 1$ surface and allowed to reach an equilibrium binding. Then, the various compounds of the data-set $(150 \mu \mathrm{M})$ were injected and their capacity to affect the binding of K8 to $\Delta \mathrm{F} 508-\mathrm{NBD} 1$ evaluated.

\subsubsection{Competition Assays}

$\mathrm{K} 8$ was used since, as a representative $\triangle \mathrm{F} 508$-CFTR chaperones, has been repeatedly used for SPR studies $[4,34,47]$ and its binding site in $\triangle$ F508-CFTR has been mapped [34]. To perform competition assays for the binding to $\triangle \mathrm{F} 508$-NBD1 between the various compounds and $\mathrm{K} 8$, the latter was resuspended at $20 \mu \mathrm{g} / \mathrm{mL}$ in $10 \mathrm{mM}$ sodium acetate $\mathrm{pH} 4.0$ and allowed to react with the first flow cell of a CM5 sensor chip pre-activated with $50 \mu \mathrm{L}$ of $0.2 \mathrm{M}$ EDC and $0.05 \mathrm{M}$ NHS and deactivated by injection of $\mathrm{HCl}-$ ethanolamine. This procedure led to the immobilization of $7800 \mathrm{RU}$ of the protein, (equal to approximately $146 \mathrm{fmol} / \mathrm{mm}^{2}$ ). Similar results were obtained for the injection of BSA $(10 \mu \mathrm{g} / \mathrm{mL})$ onto the second flow cell of each sensor chip, that was then used as a negative control and for blank subtraction. $\triangle \mathrm{F} 508-\mathrm{NBD} 1$ (at $600 \mathrm{nM}$ ) in the absence or in the presence of the various compounds (all at $500 \mu \mathrm{M}$ ) in $0.05 \%$ surfactant P20 and 5\% DMSO, pH 7.4, was injected over the K8 or BSA surfaces for $90 \mathrm{~s}$ at $10 \mu \mathrm{L} / \mathrm{min}, 25^{\circ} \mathrm{C}$ and then washed until dissociation was observed.

Supplementary Materials: Supplementary materials are available online.

Acknowledgments: This work was supported by Fondazione Italiana Fibrosi Cistica, FFC\#6/2014 to M.R., P.F. and A.O., and FCC\#7/2015 to E.M. and E.C., by the Italian Ministry of Education, University and Research (MIUR), Flagship InterOmics (cod. PB05), PRIN2015 (cod. 20157ATSLF), by Regione Lombardia-CNR, FRRB LYRA_2015-0010 Projects to L.M. and by Omics4CF CNR project to A.O. D.S. and M.D.S. have been recipient of a fellowship by Fondazione Ricerca Fibrosi Cistica.

Author Contributions: M.R., P.D. and P.F. designed and interpreted the experiments and wrote the manuscript. A.B., C.U. and M.D.S. performed and analyzed SPR experiments. D.S., A.O., G.T., E.C., performed computational studies and data analysis. E.M. prepared and contributed reagents. L.J.V.G. and L.M. contributed analytical tools.

Conflicts of Interest: The authors declare no conflict of interest.

\section{References}

1. Rogan, M.P.; Stoltz, D.A.; Hornick, D.B. Cystic fibrosis transmembrane conductance regulator intracellular processing, trafficking, and opportunities for mutation-specific treatment. Chest 2011, 139, 1480-1490. [CrossRef] [PubMed]

2. Fanen, P.; Wohlhuter-Haddad, A.; Hinzpeter, A. Genetics of cystic fibrosis: CFTR mutation classifications toward genotype-based CF therapies. Int. J. Biochem. Cell Biol. 2014, 52, 94-102. [CrossRef] [PubMed]

3. Mendes, F.; Farinha, C.M.; Felicio, V.; Alves, P.C.; Vieira, I.; Amaral, M.D. BAG-1 stabilizes mutant F508del-CFTR in a ubiquitin-like-domain-dependent manner. Cell. Physiol. Biochem. 2012, 30, 1120-1133. [CrossRef] [PubMed]

4. Colas, J.; Faure, G.; Saussereau, E.; Trudel, S.; Rabeh, W.M.; Bitam, S.; Guerrera, I.C.; Fritsch, J.; Sermet-Gaudelus, I.; Davezac, N.; et al. Disruption of cytokeratin-8 interaction with F508del-CFTR corrects its functional defect. Hum. Mol. Genet. 2012, 21, 623-634. [CrossRef] [PubMed] 
5. Le Henaff, C.; Faria Da Cunha, M.; Hatton, A.; Tondelier, D.; Marty, C.; Collet, C.; Zarka, M.; Geoffroy, V.; Zatloukal, K.; Laplantine, E.; et al. Genetic deletion of keratin 8 corrects the altered bone formation and osteopenia in a mouse model of cystic fibrosis. Hum. Mol. Genet. 2016, 25, 1281-1293. [CrossRef] [PubMed]

6. Rivas Caldas, R.; Boisrame, S. Upper aero-digestive contamination by Pseudomonas aeruginosa and implications in Cystic Fibrosis. J. Cyst. Fibros. 2015, 14, 6-15. [CrossRef] [PubMed]

7. Scott-Ward, T.S.; Amaral, M.D. Deletion of Phe508 in the first nucleotide-binding domain of the cystic fibrosis transmembrane conductance regulator increases its affinity for the heat shock cognate 70 chaperone. FEBS J. 2009, 276, 7097-7109. [CrossRef] [PubMed]

8. Amaral, M.D.; Farinha, C.M. Rescuing mutant CFTR: A multi-task approach to a better outcome in treating cystic fibrosis. Curr. Pharm. Des. 2013, 19, 3497-3508. [CrossRef] [PubMed]

9. Elborn, J.S. Cystic fibrosis. Lancet 2016, 388, 2519-2531. [CrossRef]

10. Ren, H.Y.; Grove, D.E.; De La Rosa, O.; Houck, S.A.; Sopha, P.; Van Goor, F.; Hoffman, B.J.; Cyr, D.M. VX-809 corrects folding defects in cystic fibrosis transmembrane conductance regulator protein through action on membrane-spanning domain 1. Mol. Biol. Cell 2013, 24, 3016-3024. [CrossRef] [PubMed]

11. Okiyoneda, T.; Veit, G.; Dekkers, J.F.; Bagdany, M.; Soya, N.; Xu, H.; Roldan, A.; Verkman, A.S.; Kurth, M.; Simon, A.; et al. Mechanism-based corrector combination restores DeltaF508-CFTR folding and function. Nat. Chem. Biol. 2013, 9, 444-454. [CrossRef] [PubMed]

12. Farinha, C.M.; King-Underwood, J.; Sousa, M.; Correia, A.R.; Henriques, B.J.; Roxo-Rosa, M.; Da Paula, A.C.; Williams, J.; Hirst, S.; Gomes, C.M.; et al. Revertants, low temperature, and correctors reveal the mechanism of F508del-CFTR rescue by VX-809 and suggest multiple agents for full correction. Chem. Biol. 2013, 20, 943-955. [CrossRef] [PubMed]

13. He, L.; Aleksandrov, A.A.; An, J.; Cui, L.; Yang, Z.; Brouillette, C.G.; Riordan, J.R. Restoration of NBD1 thermal stability is necessary and sufficient to correct F508 CFTR folding and assembly. J. Mol. Biol. 2015, 427, 106-120. [CrossRef] [PubMed]

14. Liu, F.; Zhang, Z.; Csanady, L.; Gadsby, D.C.; Chen, J. Molecular Structure of the Human CFTR Ion Channel. Cell 2017, 169, 85-95.e8. [CrossRef] [PubMed]

15. Hudson, R.P.; Dawson, J.E.; Chong, P.A.; Yang, Z.; Millen, L.; Thomas, P.J.; Brouillette, C.G.; Forman-Kay, J.D. Direct Binding of the Corrector VX-809 to Human CFTR NBD1: Evidence of an Allosteric Coupling between the Binding Site and the NBD1:CL4 Interface. Mol. Pharmacol. 2017, 92, 124-135. [CrossRef] [PubMed]

16. Sampson, H.M.; Robert, R.; Liao, J.; Matthes, E.; Carlile, G.W.; Hanrahan, J.W.; Thomas, D.Y. Identification of a NBD1-binding pharmacological chaperone that corrects the trafficking defect of F508del-CFTR. Chem. Biol. 2011, 18, 231-242. [CrossRef] [PubMed]

17. Lewis, H.A.; Wang, C.; Zhao, X.; Hamuro, Y.; Conners, K.; Kearins, M.C.; Lu, F.; Sauder, J.M.; Molnar, K.S.; Coales, S.J.; et al. Structure and dynamics of NBD1 from CFTR characterized using crystallography and hydrogen/deuterium exchange mass spectrometry. J. Mol. Biol. 2010, 396, 406-430. [CrossRef] [PubMed]

18. Tordai, H.; Leveles, I.; Hegedus, T. Molecular dynamics of the cryo-EM CFTR structure. Biochem. Biophys. Res. Commun. 2017, 491, 986-993. [CrossRef] [PubMed]

19. Kemp, M.M.; Weiwer, M.; Koehler, A.N. Unbiased binding assays for discovering small-molecule probes and drugs. Bioorg. Med. Chem. 2012, 20, 1979-1989. [CrossRef] [PubMed]

20. Rusnati, M.; Presta, M. Angiogenic growth factors interactome and drug discovery: The contribution of surface plasmon resonance. Cytokine Growth Factor Rev. 2015, 26, 293-310. [CrossRef] [PubMed]

21. Rusnati, M.; Bugatti, A.; Mitola, S.; Leali, D.; Bergese, P.; Depero, L.E.; Presta, M. Exploiting Surface Plasmon Resonance (SPR) Technology for the Identification of Fibroblast Growth Factor-2 (FGF2) Antagonists Endowed with Antiangiogenic Activity. Sensors 2009, 9, 6471-6503. [CrossRef] [PubMed]

22. Eckford, P.D.; Li, C.; Ramjeesingh, M.; Bear, C.E. Cystic fibrosis transmembrane conductance regulator (CFTR) potentiator VX-770 (ivacaftor) opens the defective channel gate of mutant CFTR in a phosphorylation-dependent but ATP-independent manner. J. Biol. Chem. 2012, 287, 36639-36649. [CrossRef] [PubMed]

23. Pedemonte, N.; Tomati, V.; Sondo, E.; Caci, E.; Millo, E.; Armirotti, A.; Damonte, G.; Zegarra-Moran, O.; Galietta, L.J. Dual activity of aminoarylthiazoles on the trafficking and gating defects of the cystic fibrosis transmembrane conductance regulator chloride channel caused by cystic fibrosis mutations. J. Biol. Chem. 2011, 286, 15215-15226. [CrossRef] [PubMed] 
24. Kim Chiaw, P.; Eckford, P.D.; Bear, C.E. Insights into the mechanisms underlying CFTR channel activity, the molecular basis for cystic fibrosis and strategies for therapy. Essays Biochem. 2011, 50, 233-248. [CrossRef] [PubMed]

25. Pedemonte, N.; Lukacs, G.L.; Du, K.; Caci, E.; Zegarra-Moran, O.; Galietta, L.J.; Verkman, A.S. Small-molecule correctors of defective DeltaF508-CFTR cellular processing identified by high-throughput screening. J. Clin. Investig. 2005, 115, 2564-2571. [CrossRef] [PubMed]

26. Pesce, E.; Bellotti, M.; Liessi, N.; Guariento, S.; Damonte, G.; Cichero, E.; Galatini, A.; Salis, A.; Gianotti, A.; Pedemonte, N.; et al. Synthesis and structure-activity relationship of aminoarylthiazole derivatives as correctors of the chloride transport defect in cystic fibrosis. Eur. J. Med. Chem. 2015, 99, 14-35. [CrossRef] [PubMed]

27. Wang, C.; Protasevich, I.; Yang, Z.; Seehausen, D.; Skalak, T.; Zhao, X.; Atwell, S.; Spencer Emtage, J.; Wetmore, D.R.; Brouillette, C.G.; et al. Integrated biophysical studies implicate partial unfolding of NBD1 of CFTR in the molecular pathogenesis of F508del cystic fibrosis. Protein Sci. 2010, 19, 1932-1947. [CrossRef] [PubMed]

28. Protasevich, I.; Yang, Z.; Wang, C.; Atwell, S.; Zhao, X.; Emtage, S.; Wetmore, D.; Hunt, J.F.; Brouillette, C.G. Thermal unfolding studies show the disease causing F508del mutation in CFTR thermodynamically destabilizes nucleotide-binding domain 1. Protein Sci. 2010, 19, 1917-1931. [CrossRef] [PubMed]

29. Rabeh, W.M.; Bossard, F.; Xu, H.; Okiyoneda, T.; Bagdany, M.; Mulvihill, C.M.; Du, K.; di Bernardo, S.; Liu, Y.; Konermann, L.; et al. Correction of both NBD1 energetics and domain interface is required to restore DeltaF508 CFTR folding and function. Cell 2012, 148, 150-163. [CrossRef] [PubMed]

30. Hudson, R.P.; Chong, P.A.; Protasevich, I.I.; Vernon, R.; Noy, E.; Bihler, H.; An, J.L.; Kalid, O.; Sela-Culang, I.; Mense, M.; et al. Conformational changes relevant to channel activity and folding within the first nucleotide binding domain of the cystic fibrosis transmembrane conductance regulator. J. Biol. Chem. 2012, 287, 28480-28494. [CrossRef] [PubMed]

31. Wieczorek, G.; Zielenkiewicz, P. DeltaF508 mutation increases conformational flexibility of CFTR protein. J. Cyst. Fibros. 2008, 7, 295-300. [CrossRef] [PubMed]

32. Callebaut, I.; Hoffmann, B.; Lehn, P.; Mornon, J.P. Molecular modelling and molecular dynamics of CFTR. Cell. Mol. Life Sci. 2017, 74, 3-22. [CrossRef] [PubMed]

33. Kim Chiaw, P.; Huan, L.J.; Gagnon, S.; Ly, D.; Sweezey, N.; Rotin, D.; Deber, C.M.; Bear, C.E. Functional rescue of DeltaF508-CFTR by peptides designed to mimic sorting motifs. Chem. Biol. 2009, 16, 520-530. [CrossRef] [PubMed]

34. Odolczyk, N.; Fritsch, J.; Norez, C.; Servel, N.; da Cunha, M.F.; Bitam, S.; Kupniewska, A.; Wiszniewski, L.; Colas, J.; Tarnowski, K.; et al. Discovery of novel potent DeltaF508-CFTR correctors that target the nucleotide binding domain. EMBO Mol. Med. 2013, 5, 1484-1501. [CrossRef] [PubMed]

35. Zhenin, M.; Noy, E.; Senderowitz, H. REMD Simulations Reveal the Dynamic Profile and Mechanism of Action of Deleterious, Rescuing, and Stabilizing Perturbations to NBD1 from CFTR. J. Chem. Inf. Model. 2015, 55, 2349-2364. [CrossRef] [PubMed]

36. Giorgio, C.; Russo, S.; Incerti, M.; Bugatti, A.; Vacondio, F.; Barocelli, E.; Mor, M.; Pala, D.; Hassan-Mohamed, I.; Gioiello, A.; et al. Biochemical characterization of EphA2 antagonists with improved physico-chemical properties by cell-based assays and surface plasmon resonance analysis. Biochem. Pharmacol. 2016, 99, 18-30. [CrossRef] [PubMed]

37. Peters, K.W.; Okiyoneda, T.; Balch, W.E.; Braakman, I.; Brodsky, J.L.; Guggino, W.B.; Penland, C.M.; Pollard, H.B.; Sorscher, E.J.; Skach, W.R.; et al. CFTR Folding Consortium: Methods available for studies of CFTR folding and correction. Methods Mol. Biol. 2011, 742, 335-353. [PubMed]

38. Moran, O.; Galietta, L.J.; Zegarra-Moran, O. Binding site of activators of the cystic fibrosis transmembrane conductance regulator in the nucleotide binding domains. Cell. Mol. Life Sci. 2005, 62, 446-460. [CrossRef] [PubMed]

39. Chong, P.A.; Farber, P.J.; Vernon, R.M.; Hudson, R.P.; Mittermaier, A.K.; Forman-Kay, J.D. Deletion of Phenylalanine 508 in the First Nucleotide-binding Domain of the Cystic Fibrosis Transmembrane Conductance Regulator Increases Conformational Exchange and Inhibits Dimerization. J. Biol. Chem. 2015, 290, 22862-22878. [CrossRef] [PubMed] 
40. Lewis, H.A.; Buchanan, S.G.; Burley, S.K.; Conners, K.; Dickey, M.; Dorwart, M.; Fowler, R.; Gao, X.; Guggino, W.B.; Hendrickson, W.A.; et al. Structure of nucleotide-binding domain 1 of the cystic fibrosis transmembrane conductance regulator. EMBO J. 2004, 23, 282-293. [CrossRef] [PubMed]

41. Mendoza, J.L.; Schmidt, A.; Li, Q.; Nuvaga, E.; Barrett, T.; Bridges, R.J.; Feranchak, A.P.; Brautigam, C.A.; Thomas, P.J. Requirements for efficient correction of DeltaF508 CFTR revealed by analyses of evolved sequences. Cell 2012, 148, 164-174. [CrossRef] [PubMed]

42. Webb, B.; Sali, A. Protein Structure Modeling with MODELLER. In Functional Genomics; Kaufmann, M., Klinger, C., Savelsbergh, A., Eds.; Methods in Molecular Biology; Humana Press: New York, NY, USA, 2017; Volume 1654, pp. 39-54. [CrossRef]

43. Dundas, J.; Ouyang, Z.; Tseng, J.; Binkowski, A.; Turpaz, Y.; Liang, J. CASTp: Computed atlas of surface topography of proteins with structural and topographical mapping of functionally annotated residues. Nucleic Acids Res. 2006, 34, W116-W118. [CrossRef] [PubMed]

44. Morris, G.M.; Huey, R.; Lindstrom, W.; Sanner, M.F.; Belew, R.K.; Goodsell, D.S.; Olson, A.J. AutoDock4 and AutoDockTools4: Automated docking with selective receptor flexibility. J. Comput. Chem. 2009, 30, 2785-2791. [CrossRef] [PubMed]

45. Trott, O.; Olson, A.J. AutoDock Vina: Improving the speed and accuracy of docking with a new scoring function, efficient optimization, and multithreading. J. Comput. Chem. 2010, 31, 455-461. [CrossRef] [PubMed]

46. Shrake, A.; Rupley, J.A. Environment and exposure to solvent of protein atoms. Lysozyme and insulin. J. Mol. Biol. 1973, 79, 351-371. [CrossRef]

47. Premchandar, A.; Kupniewska, A.; Bonna, A.; Faure, G.; Fraczyk, T.; Roldan, A.; Hoffmann, B.; Faria da Cunha, M.; Herrmann, H.; Lukacs, G.L.; et al. New insights into interactions between the nucleotide-binding domain of CFTR and keratin 8. Protein Sci. 2017, 26, 343-354. [CrossRef] [PubMed]

Sample Availability: Samples of compounds 4-7 can be available from the author Enrico Millo.

(C) 2018 by the authors. Licensee MDPI, Basel, Switzerland. This article is an open access article distributed under the terms and conditions of the Creative Commons Attribution (CC BY) license (http://creativecommons.org/licenses/by/4.0/). 